\title{
El aprendizaje colaborativo en la enseñanza de la Matemática a nivel de pregrado
}

Collaborative learning in the teaching of mathematics at the undergraduate level

\section{JUAN CARLOS LUNA SANTOS ${ }^{1}$}

https://orcid.org/0000-0001-8675-6850

\begin{abstract}
SERESUMEN: El aprendizaje colaborativo, es hoy un factor clave en el desarrollo de la enseñanza de la Matemática y su estudio interviene en el progreso de la calidad educativa. Por lo anterior nuestro estudio tiene por propósito analizar de qué manera el aprendizaje cola-borativo se emplea en la enseñanza de las matemáticas a través de la solución de ejerci-cios y el hacer uso de estrategias. Se realizó una aproximación conceptual de diferentes autores que ayudan a evidenciar la importancia que tiene, en la organización social en el aula estableciendo una interdependencia positiva. La información me permitió concluir que, como estrategia en Matemática a nivel pregrado, incrementa la productividad aca-démica de los alumnos. Sin embargo, es necesario que se desarrolle como estrategia en las universidades, y si se complementara con el uso de las TIC generaría un aprendizaje muy beneficioso.
\end{abstract}

Palabras clave: aprendizaje colaborativo; cognitivo; procedimental.

ABSTRACT: Collaborative learning is today a key factor in the development of the teaching of Mat-hematics and its study intervenes in the progress of educational quality. Therefore, our study aims to analyze how collaborative learning is used in the teaching of mathematics through the solution of exercises and the use of strategies. A conceptual approach was made by different authors that help to show the importance it has, in the social organization in the classroom, establishing a positive interdependence. The information allowed me to conclude that, as a strategy in Mathematics at the undergraduate level, it increases the academic productivity of students. However, it needs to be developed as a strategy in universities, and if it were complemented with the use of ICT, it would generate very beneficial learning.

Keywords: Collaborative Learning; Cognitive; Procedural. 


\section{INTRODUCCIÓN}

El aprendizaje colaborativo es una técnica que impulsa el estudio de los alumnos, que se fundamenta en la labor de pequeñas asociaciones de estudiantes que se juntan en las clases, con el propósito de desarrollar sus habilidades, coordinar entre ellos; utilizando el diálogo, donde se respetan sus puntos de vista, se hace uso de niveles de aprendizaje que se integran para enriquecer su entendimiento. Es una táctica pedagógica cuyo propósito es fomentar y estructurar la intersubjetividad a través de la relación de los alumnos donde se forman equipos de trabajo, cuyo propósito es aprender en el aula, esta interacción hace que al trabajar juntos puedan mejorar su aprendizaje y su desarrollo personal (Rivero \& Suárez, 2017). Durante muchos siglos antes se hablaba de las grandes privilegios y beneficios que tiene la agrupación de los alumnos universitarios en el aula, para desarrollar aspectos académicos llamadas tareas, mediante esta técnica se observa mejoras en sus aprendizajes de forma significativa (Ramirez,2017) Con la aplicación del Aprendizaje Colaborativo como estrategia de enseñanza se busca propiciar cambios en el modelo de enseñanza aprendizaje, de uno centrado en el docente a un modelo centrado en el estudiante, a fin de desarrollar roles y responsabilidades en ambos y ayuden al propósito de los logros entendiendo a este como un proceso cognitivo, procedimental, y actitudinal (Rivadeneira-Silva, 2017), ellos dejarían de ser netamente receptores de la información transmitidas por sus docentes y se volverían en cambio en "personas participativas en el proceso de su aprendizaje" (Danker, 2015). Para ello, se busca que la enseñanza colaborativa se utilice en la solución de los ejercicios de forma analítica, ayudando a eliminar el rechazo que se tiene por esta ciencia exacta, al tiempo que ayuda a satisfacer los requerimientos de la UNESCO acerca del desarrollo y aprendizaje de las ciencias exactas.

No es casualidad que durante muchos años las dudas que presentan los alumnos al afrontar problemas académicos de ciencias, cuando resuelven ejercicios matemáticos en las universidades, constituye una gran problemática, es así como nos planteamos: ¿Cómo el aprendizaje colaborativo se emplea en el estudio de la matemática a nivel de pregrado? ¿Cómo contribuye el aprendizaje colaborativo en el estudio de la Matemática a nivel de pregrado en los aspectos cognitivos y procedimentales? Por ello, nos planteamos ya sea porque desconocen los temas, no tienen una base sólida del tema tratado o porque sus profesores no los han sabido motivar desde su formación escolar. Esta problemática se ha ido acrecentando a través de los años, y q hasta la fecha no se ha podido resolver, a pesar que se busca alternativas de solución. Ante esta perspectiva presentamos algunas alternativas a desarrollar como es el caso de estrategias para el estímulo del diálogo utilizando nomenclaturas, la escucha del otro y la evaluación recíproca procedimental.

Frente a este reto, se intentó renovar e innovar, utilizando estrategias de aprendizaje. Para la aplicación se hizo uso del aprendizaje colaborativo que busca en todos los casos, que los alumnos aprendan de manera grupal, se integren socialmente para que les resulte más fácil asimilar conocimientos y poder utilizarlos constituyéndose una propuesta de un alto alcance para el aprendizaje en la educación. En el sector educacional durante los últimos periodos de tiempo, en cada año transcurrido las estrategias metodológicas se van innovando de acuerdo a las eventualidades presentadas en el aula. Se pretende mejorar nuestro sistema educativo haciendo uso de una estrategia colaborativa donde el protagonista principal es el estudiante. Esta estrategia trata de convertir a los alumnos en participes activos 
de sus propios conocimientos y que para ello se busca que el estudio colaborativo se utilice convenientemente en el proceso de su estudio. Además, se trata de cambiar el rechazo a las matemáticas, motivando a los alumnos para su aceptación. Tratando de entender la problemática y buscar alternativas de solución.

Este estudio busca un diagnóstico de cómo se encuentra su rendimiento académico, realizar propuestas que mejoren su logro del estudio en los alumnos universitarios. Además, reestructurar e innovar la planeación académica y administrativa de los planes de estudio vinculadas a integrar la Ley Nueva Universitaria en beneficio de los jóvenes universitarios.

Una herramienta a considerar es la colaboración, utilizada en el aula de clases donde se desarrollan dinámicas provocan mejores interacciones entre los alumnos y nos enseñan a superar problemas académicos, buscando el aumento de un aprendizaje de orden superior. Este estudio pretende, que el aprendizaje colaborativo sea utilizado en las aulas escolares de manera obligatoria ya que dicha estrategia desarrolla el valor de las relaciones interpersonales en grupos al considerarse como valor para la formación del estudiante. Asimismo, esta investigación pretende contribuir al desarrollo de los estudiantes universitarios y a su formación, mejorando sus calificaciones. Con esta técnica se benefician los estudiantes en su entorno ya que promueve valores como el compromiso de la comunicación la solidaridad y el trabajo en equipo.

En tal sentido, se plantea el siguiente objetivo: analizar de qué manera la enseñanza colaborativa se emplea en el estudio de la Matemática en los aspectos cognitivos y procedimentales.

Se entiende que el estudiante, se beneficia notablemente en la enseñanza de la Matemática al hacer uso del aprendizaje colaborativo, ya que este los impulsa a desarrollar metas, dentro del salón de clases, donde a se asumen responsabilidades en forma grupal, al momento de relacionarse con los demás, respetando y aceptando sus puntos de vista, exponiendo sus criterios de forma analítica y razonada. Además, se observa que los estudiantes, se motivan y comprenden con la ayuda de sus pares. Cuando trabajan en grupos reciben aportes de sus compañeros y se sociabilizan de una manera más didáctica y asertiva. Esta investigación también investiga cómo aporta el aprendizaje colaborativo, en la formación académica y la práctica docente de los alumnos, como en la investigación cualitativa como una manera de corroborar de qué manera se utilizaba estrategias didácticas, cómo se obtuvieron los datos directos y concretos entre el investigador y el objeto estudiado, lo que permitió profundizar las interacciones de las personas y en sus apreciaciones con el uso del aprendizaje colaborativo.

Además, el estudio busca desarrollar las relaciones interpersonales en grupos al considerarlo como valor para la educación del alumno. Para diseñar las sesiones de clases, de incluyen temas específicos que requerían de resolver problemas donde cada pregunta tenía un tiempo y valor específico por pregunta, compara en esta actividad la forma individual y grupal de trabajar, cuyo objetivo fue desarrollar los aspectos cognitivos y procedimentales, la interacción grupal a través del trabajo colaborativo, obteniendo un resultado concluyente que reflejaba cómo esta estrategia mejoraba su aprendizaje considerablemente cuando se trabaja en grupo delegando a la forma individual. 
Lo más probable de esta investigación es que se haga uso de investigaciones acerca del aprendizaje colaborativo de una manera experimental donde se conformaron muestras individuales y grupales monitoreadas y estudiadas para dar a conocer si realmente se mejora el aprendizaje en las matemáticas y si se desarrollan habilidades en los estudiantes donde el razonamiento lógico la deducción cognitiva es importante en el desarrollo de las operaciones matemáticas a todo esto se le agrega que ellos deberán afrontar situaciones diversas al interrelacionarse y que a pesar de todas estas consideraciones el resultado del aprendizaje sea muy beneficioso, además se debe de considerar que al momento de comparar quienes utilizaron este aprendizaje colaborativo y quienes no se obtiene como resultado que aquellos que lo utilizaron de forma grupal obtuvieron mejores resultados

De acuerdo con el Proyecto Tuning América Latina y las destrezas a desarrollar en la adiestramiento del matemático al realizar un estudio en este tema, me permitirá analizar diversas estrategias metodológicas de aprendizaje colaborativo elegir cuales son la más propicias a utilizar en el aprendizaje de los estudiantes, también resultará propicio realizar un diagnóstico como se encontraba en esos momentos el rendimiento académico y de qué manera lo realizaban para luego presentar propuestas que mejoren el aprendizaje en los estudiantes. Por tanto, se afirma que el aprendizaje colaborativo en el estudio de la Matemática a nivel de pregrado mejora estratégicamente la enseñanza de los estudiantes.

De las experiencias realizadas también se desliga que la sinergia entre el AC y en el estudio de las matemáticas en las universidades mejora beneficiosamente el clima de trabajo en el aula, impulsa de forma efectiva la adquisición nuevas competencias, aumenta el interés de los jóvenes universitario sobre las materias en estudio, y obtiene mejores calificaciones académicas.

\section{DESARROLLO}

\section{El aprendizaje colaborativo se emplea en la enseñanza de la Matemática a nivel de pregrado.}

De manera análoga se afirma que el estudiante se beneficia notablemente en la enseñanza de la matemática al momento de emplear el aprendizaje colaborativo, ya que este los impulsa a desarrollar acciones dentro del aula donde asumen compromisos en forma grupal relacionándose con los demás, respetando y aceptando sus puntos de vista, exponiendo sus criterios de forma analítica y razonada, manifiesta que los alumnos del se motivan más cuando trabajan en grupos reciben aportes de sus compañeros, se sociabilizan de una manera más didáctica y asertiva. De igual modo, con la finalidad de investigar cómo aporta el aprendizaje colaborativo, en la formación académica y la práctica docente de los alumnos, como en la investigación cualitativa, como una manera de corroborar de qué manera se utilizaba, aplicaron ellos una didáctica, donde obtuvieron datos directos y concretos entre el investigador y el objeto estudiado, lo que permitió profundizar las interacciones de las personas y en sus apreciaciones con el uso del aprendizaje colaborativo. De manera semejante nos menciona además que al estudiar acerca del trabajo cooperativo y colaborativo muestra muchas incidencias en el estudio de las matemáticas, es un gran reto donde se ha llevado a cabo la resolución de talleres y el trabajo en equipo de forma grupal o individual 
para poder comparar utilizar diversas técnicas al proponer este tema involucra capacidades en la enseñanza de las matemáticas obteniéndose un mejor resultado cuando se trabaja en grupo colaborativamente que en forma individual, de esta forma cuando se involucran todos los estudiantes se incurre adecuadamente en el desarrollo de sus habilidades. Señala que el aprendizaje colaborativo es indispensable para el carácter formativo educacional en los diversos entornos estudiantiles. Aquí se menciona que los alumnos de economía al conformar grupos para realizar tareas de los cursos se complementan sustancialmente y aprenden significativamente (Yong Chang et al., 2018).

Del mismo modo se consideró, que este estudio busque desarrollar las relaciones interpersonales en grupos al considerarlo como valor central para la educación. De esta forma el aprendizaje colaborativo fortalece el pensamiento crítico en la enseñanza de las matemáticas, la labor en grupos es tan eficaz que impulsa el incremento de habilidades en los alumnos en su entorno universitario enseñándoles a convivir y relacionarse a pesar de las divergencias de caracteres que se presentan en la labor por agrupación, impulsando su adaptación hacia objetivos y fines que resultan beneficiosos en la resolución de problemas. Nos menciona que esta investigación sobre los cursos virtuales en la Universidad Minuto de Dios, hizo uso de las bitácoras de observación, y cuestionarios aplicados a los alumnos. Por ello, demuestra que estos alumnos agrupados en equipos, utilizando diferentes métodos de aprendizaje obtienen mejores resultados académicos, una mayor integración y los objetivos trazados se cumplen considerablemente (Guerra Santana et al., 2019).

Esta estrategia al ser utilizada mejora y complementa considerablemente la asimilación de conocimiento al momento de resolver problemas en el aula, también debemos de considerar el uso de las TIC que fortalecen su aprendizaje obteniendo resultados muy óptimos que favorecen el aprendizaje y los motiva a aprender cada día más. Acorde con lo realizado por la Universidad Estatal a Distancia (UNED) de Costa Rica allí se aplicó un sondeo a dos grupos de estudiantes, cada uno concerniente a dos cursos virtuales donde se utilizó el aprendizaje colaborativo como herramientas con preguntas abiertas y cerradas. Por último, los estudiantes respondieron que prefieren el trabajo colaborativo, porque complementa sus conocimientos al momento de interactuar entre los compañeros de aula y si utilizan las herramientas virtuales se hace más eficaz su aprendizaje, en este caso dependerá del estilo de aprendizaje que asuma el docente, de la manera cómo motiva la clase, de las herramientas que utiliza, para hacer que los alumnos universitarios aprendan de la mejor manera., pudiéndose verificar que el aprendizaje colaborativo contribuye en la enseñanza de la Matemática.

Nos menciona, además, al presentar los cuestionarios con un conjunto de problemas clasificados por temas desarrollados y con características para poderlas resolver las operaciones matemáticas, a nivel universitario muchos sintieron dudas para su resolución cuando resolvieron individualmente, pero al integrarse en grupo y utilizar como estrategia en el aprendizaje colaborativo se fortalecieron académicamente a tal punto que se motivaron por investigar y aprender intercambiar opiniones, aprender de los demás unificar ideas y obtener como resultado un aprendizaje significativo que les hizo aprender en conjunto así poder mejorar su calificación.

Este estudio nos menciona que participaron los estudiantes universitarios panameños donde se les aplica el instrumento llamado cuestionario y una ficha de observación. Asimismo, 
los estudiantes demostraron poder resolver los problemas, acomodar y estructurar los datos matemáticos, así como auto promoverse y exhibir una intervención dinámica en el crecimiento de las actividades educativas de la clase. Acorde con que realizaron investigaciones acerca del aprendizaje colaborativo de una manera experimental conformando muestras individuales y grupales monitoreadas y estudiadas para conocer si realmente se mejora el aprendizaje en las matemáticas y si se desarrollan habilidades en los estudiantes de forma memorística y procedimental al hacer uso de cálculo y algoritmos en la matemática, considerando además desafiar situaciones problemáticas derivadas de las relaciones personales arrojando como resultado que el aprendizaje colaborativo es muy beneficioso y ventajoso con respecto a aquellos que no utilizaron esta estrategia (Morales-Maure et al., 2018).

\section{El aprendizaje colaborativo contribuye en la enseñanza de la matemática en los aspectos cognitivos y procedimentales como se afirma de manera análoga}

De igual modo con lo que se menciona sabemos que si el aprendizaje colaborativo se complementa con el Flipped Classroom, esta herramienta contribuye notablemente a resolver y asimilar conocimientos en los alumnos universitarios sobre todo en la resolución de problemas matemáticos, inclusive les permite la entrega de trabajos académicos de calidad y una resolución relativamente ordenada y precisa consultar a medios informáticos y analizar videos tutoriales del tema específico. Mejorando considerablemente y participando activamente de los trabajos académicos. De igual manera el intercambio cultural social y de ideas, fomentará el aprendizaje, nos apoya tal aseveración la teoría de aprendizaje de Vygotsky. Además, se asume que el estudio colaborativo con el Fllipped Classroom tienen mucho más sentido de aprendizaje y son mejores coordinados que el desarrollado por la clase invertida (Sánchez-Cruzado et al., 2018). Del mismo modo concordamos con lo expresado en que es de mucha necesidad darle un valor significativo a la sesión de aprendizaje desarrollados en el aula universitaria donde las interacciones y experiencias que se generan en este contexto son significativas, esenciales en el aprendizaje donde el uso de las tecnologías conjuntamente con el empleo del aprendizaje colaborativo complementa que el alumno asimile los conocimientos integralmente.

De manera análoga nos menciona además que el uso de las TIC desarrolla habilidades y destrezas en los estudiantes, constituye una alternativa de ayuda para los estudiantes universitarios que tienen problemas con el aprendizaje de las matemáticas ya que les ayuda a interactuar de manera organizada aprendiendo e intercambiando información en el área de ciencia de este modo se busca complementar los conocimientos proporcionados por los docentes y mejorar su aprendizaje. Nos menciona que es un problema las calificaciones académicas de los alumnos en el curso de Matemática donde tiene un elevado porcentaje de reprobación, se desaprueba el curso y otros optan por la deserción. Por ello se ha optado como propuesta el uso de la enseñanza colaborativo y el uso de las TIC, con mejoras educativas para el desarrollo del curso en el aula y potenciar las habilidades en los alumnos donde le proporcione tener mayor autonomía, predisposición y trabajo cooperativo. Para comprender mejor en una de las fases desarrolladas por el autor es elegir recursos tecnológicos donde se integran y gestionan datos ocasionando la interacción, donde se observa que la enseñanza y motivación en la solución de problemas matemáticos en forma de cuestionarios (Montes en al., 2018). 
Del mismo modo si tomamos como punto de partida el factor pedagógico, lo que influye en la productividad académico de los alumnos según el cuestionario que sirvió como información, constituyéndose como una de las variables obtenidas el tiempo de dedicación al estudio, la manera y el tipo de estrategia utilizada, siendo la propuesta en nuestra investigación la más recomendada. Los resultados que se reportan, en la productividad académica de los estudiantes de ingeniería de la FUAC, en el transcurso de los tres primeros semestres de su formación académica profesional son progresivos cuando se hace uso de estrategias que ayudan al aprendizaje.

Similarmente nos menciona además, acerca del rendimiento académico en el estudio de la Matemática a nivel universitario, la problemática que presenta y la propuesta de brindar una innovadora forma de enseñar, originando que los estudiantes aprendan utilizando estrategias, donde los conocimientos adquiridos deberán de ser empleados en el desarrollo de las tareas académicas evidenciándose avances en los aspectos cognitivo y procedimental, constituyéndose como un éxito académico (Camacho et al., 2017). Acorde con que existe relación entre el aprendizaje colaborativo utilizado en los cursos virtuales y las diversas maneras de enseñanza en estudiantes en formación universitario han ido tomados con gran importancia a través de los años en los ambientes virtuales. Donde la estrategia empleada en un determinado momento se convierte en una interacción social mediante la cual alumnos y docentes se relacionan fortaleciendo notablemente el aprendizaje.

Conforme a que esta investigación sobre los cursos virtuales en la Universidad Minuto de Dios, hizo uso de las bitácoras de observación, y cuestionarios aplicados a los alumnos. Por ello nos demuestra que estos alumnos agrupados en equipos, utilizando diferentes métodos de aprendizaje obtienen mejores resultados académicos, una mayor integración y los objetivos trazados se cumplen considerablemente, donde demuestran sus capacidades y destrezas utilizándolo en la solución de problemas.

Similarmente nos menciona además que el aprendizaje colaborativo fortalece el pensamiento crítico en la enseñanza de las matemáticas, la labor en grupos es tan eficaz que impulsa el desarrollo de capacidades en los alumnos en su entorno universitario, enseñándoles a convivir y relacionarse a pesar de las divergencias de caracteres que se presentan en la labor por agrupación, impulsando su adaptación hacia objetivos y fines que resultan beneficiosos en la resolución de problemas (Arenas, 2017).

La metodología colaborativa utilizada por las redes sociales se sugiere utilizar ya que desarrolla en los universitarios habilidades y destrezas en la solución de ejercicios matemáticos, independientes del género. Por tanto, los estudiantes muestran un alto nivel de satisfacción hacia la estrategia porque, en un área tan abstracta, le permite mejorar sus niveles de aprendizaje, siendo accesible para las diferentes actividades. La utilización de esta propuesta fue valorada y reconocida por los padres de familia como una forma muy positiva para la enseñanza. De manera semejante nos comentan que las estrategias que se utilizan en la enseñanza aprendizaje son de gran alcance para la solución de problemas en las matemáticas. Además, que el uso de las TIC y las redes sociales suman considerablemente, creándose entornos que son propicios para el aprendizaje de los estudiantes participantes en este contexto. Del mismo modo, hace saber cómo el aprendizaje colaborativo se emplea beneficiosamente en las matemáticas conociendo que ellas utilizan un lenguaje simbólico, 
representativo e interpretativo llamado también vocabulario técnico, necesario para descifrar símbolos, notaciones científicas, además se debe de proponer el desarrollo de las competencias matemáticas como finalidad de su enseñanza (Cruz et al., 2017).

Acorde con el autor que propone una metodología activa que fomente en los estudiantes el trabajo en equipo y una participación activa en el salón de clases. Promoviendo en todo momento la labor del grupo para alcanzar un mayor logro de las destrezas en los estudiantes al momento de resolver ejercicios de matemática reconociendo que se fomenta un ambiente de efectividad para buscar un buen clima en el aula, se promueve la escucha activa en el aula cuya consigna es: "compartan y apoyen mutuamente" en el desarrollo de los trabajos en equipo y que el esfuerzo desplegado tenga como meta la entrega del trabajo académico mejor redactado y complementado, del mismo modo comenta que en esta investigación se benefician del aprendizaje colaborativo los estudiantes considerablemente, donde hace saber que la estrategia que utilice el maestro, determinará el aprendizaje en la resolución de los problemas en la matemática en los estudiantes universitarios y que estos sean significativo para ellos. O sea, menciona, además, que presentan variables independientes y dependientes que dan apoyo a la proposición que se plantea como hipótesis de la investigación que se indica al hacer uso de estrategias didácticas para mejorar el proceso de estudio en la enseñanza de la Matemática obteniéndose los resultados esperados medidos mediante indicadores desarrollados y verificándose que es beneficioso para los estudiantes universitarios (Torres, 2018).

Acorde con que el aprendizaje colaborativo beneficia en la aprendizaje de la Matemática a los universitarios si se utilizan estrategias adecuadas se mencionan: la gamificación es decir el uso de juegos en contexto de índole distinto quienes cuentan con elementos comunes como las mecánicas, las dinámicas y las motivacionales la segunda estrategia es el aprendizaje basado en juegos que se refiere al uso de los juegos en ambientes adecuados y orientados a la formación educativa, cuya intención es utilizar una parte de su tiempo en propósitos educativos, de esta forma el alumno aprende a desenvolverse en el aula respetando las normas de interactividad y realimentación. Asimismo, comenta que el manejo de las TIC por parte de los estudiantes en la actualidad juega un papel principal en el estudio de las matemáticas, al utilizar este instrumento, todo ello mejora innovadoramente su aprendizaje. Finalmente, menciona además acerca de los aspectos procedimentales que debemos de abordar para el aprendizaje de las matemáticas como es el desarrollo y conocimiento de nuevas tecnologías muchas veces en forma de juegos interactivos que incentivan y mejoran el aprendizaje de los alumnos en grupos (Zabala-Vargas, 2020).

\section{CONCLUSIONES}

Se confirma que, al emplearse el aprendizaje colaborativo como estrategia en la enseñanza de la Matemática a nivel de pregrado, mejora estratégicamente en la enseñanza de los estudiantes.

También, que al emplearse el aprendizaje colaborativo como estrategia en la enseñanza de la Matemática y que, al desarrollarse, en los aspectos cognitivos y procedimentales en los estudiantes, fortalece su aprendizaje significativamente. 
Nuestra propuesta a futuro es utilizar el aprendizaje colaborativo en la enseñanza de la Matemática a nivel de pregrado de una manera estratégica y obligatoria, complementándolo, con la capacitación al docente para utilizar nuevas tecnologías digitales, todo esto contribuirá en el mejoramiento de su aprendizaje en el estudiante de pregrado.

\section{REFERENCIAS}

Arenas, C. (2017). Los estilos de aprendizaje y su relación con el aprendizaje colaborativo en cursos virtuales. [Tesis de Maestría, Tecnológico de Monterrey]. Repositorio Institucional. https://repositorio.tec.mx/bitstream/handle/11285/622384/TESIS\%20 RITEC.pdf? sequence $=1 \&$ isAllowed $=\mathrm{y}$

Cruz, I., Puentes, A., \& Cabero, J. (2017). The use of social networks for the teaching of mathematics. Revista Espacios, 38(55). https:/idus.us.es/bitstream/ handle/11441/67242/a17v38n55p31.pdf?sequence=1\%20\&isAllowed=y

Danker, B. (2015). Using Flipped Classroom Approach to Explore Deep Learning in Large Classrooms. Revista Journal of Education, 3(1), 171-186. https://files.eric.ed.gov/ fulltext/EJ1100618.pdf

Díaz, L., Sarmiento, H., \& Rodríguez, D. (2017). Relación entre el Rendimiento Académico en Matemáticas y los Estilos de Aprendizaje de los Estudiantes de la Fundación Universidad Autónoma de Colombia. Revista de Estilos de Aprendizaje, 10(20), 3462. http://revistaestilosdeaprendizaje.com/article/download/1056/1770/

Guerra, M., Rodríguez, J., \& Rodríguez, A. (2019). Aprendizaje colaborativo: experiencia innovadora en el alumnado universitario. Revista de estudios y experiencias en educación, 18(36), 269-281. https://scielo.conicyt.cl/pdf/rexe/v18n36/0718-5162rexe-18-36-269.pdf

Montes, R., Escobar, M., y Cadavid, G. (2018). Uso de herramientas tecnológicas en el desarrollo de un curso de Matemáticas 1 en la Universidad Tecnológica de Pereira. Revista entre Ciencia e Ingeniería, 12(23), 66-71. http://www.scielo.org.co/pdf/ecei/ v12n23/1909-8367-ecei-12-23-00066.pdf

Morales, L., García, O., Torres, A., \& Lebrija, A. (2018). Habilidades cognitivas a través de la estrategia de aprendizaje cooperativo y perfeccionamiento epistemológico en Matemática de estudiantes de primer año de Universidad. Revista Formación Universitaria, 11(2), 45-56. https://scielo.conicyt.cl/pdf/formuniv/v11n2/07185006-formuniv-11-02-00045.pdf

Ramírez, S. (2017). El aprendizaje colaborativo y su influencia en el logro del aprendizaje en el Curso de Contabilidad de Instituciones Financieras de una Universidad Pública de la Región Huánuco. [Tesis de Maestría, Universidad Peruana Cayetano Heredia]. Repositorio Institucional. http://repositorio.upch.edu.pe/bitstream/handle/ upch/1450/Aprendizaje_RamirezRengifo_Segundo.pdf?sequence=1\&isAllowed=y

Rivadeneira, E., \& Silva, R. (2017). Aprendizaje Basado en la Investigación en el Trabajo Autónomo y en Equipo. Revista Científica Electrónica de Ciencias Gerenciales, 13(38), 5-16. https://www.redalyc.org/pdf/782/78253678001.pdf 
Rivero, C., \& Suárez C. (2017). Mobile Learning y el Aprendizaje de las Matemáticas: el caso del Proyecto Mati-Tec1 en el Perú. Revista Tendencias Pedagógicas, 30(2), 3752. https://dialnet.unirioja.es/descarga/articulo/6164820.pdf

Sánchez, C., Sánchez, T., \& Macías, J. (2018). Flipped classroom como estrategia metodológica para mejorar la competencia en trabajo grupal en didáctica de la matemática. Revista Matemáticas, Educación y Sociedad, 1(3), 31-43. http:// mesjournal.es/ojs/index.php/mes/article/download/18/23/

Torres C. (2018). Estrategias de gestión del docente en el aula para mejorar el aprendizaje colaborativo de los estudiantes del $V$ ciclo de educación de la especialidad de primaria de la universidad nacional "Pedro Ruiz Gallo", Lambayeque, 2018. [Tesis de Maestría, Universidad Nacional Pedro Ruiz Gallo]. Repositorio Institucional. http://repositorio.unprg.edu.pe/bitstream/handle/UNPRG/7276/BC- $\quad 2103 \% 20$ TORRES\%20VIDARTE.pdf?sequence=1\&isAllowed=y

Yong, E., Cedeño, E., Tubay, M., \& Cedeño, L. (2018). Trabajo colaborativo y el aprendizaje de las matemáticas en los alumnos de Economía de la UTEQ. Revista Ciencia e Investigación, 3(10), 10-15. https://revistas.utb.edu.ec/index.php/sr/article/ download/271/350/

Zabala, S., Ardila, D., García, L., \& Benito, B. (2020). Aprendizaje Basado en Juegos (GBL) aplicado a la enseñanza de la matemática en Educación Superior. Una revisión sistemática de literatura. Revista Formación universitaria, 13(1), 13-26. https://scielo.conicyt.cl/pdf/formuniv/v13n1/0718-5006-formuniv-13-01-13.pdf 